\title{
TUBERCULOSIS AT AN UNUSUAL SITE
}

\author{
ZAHIDUL HAQ ${ }^{1}$, MD. SHAHRIAR MAHBUB ${ }^{2}$, MD TITU MIAH $^{3}$, MD BILLAL ALAM ${ }^{4}$, RIAZ AHMED \\ CHOWDHURY $^{5}$, HAMNAZMUL AHASAN ${ }^{6}$
}

\begin{abstract}
Tuberculosis involving the anorectal region is a rare extrapulmonary form of the disease. We report two cases of isolated rectal tuberculosis with different presentations. On both instances, diagnosis was confirmed by histological examination and treated with anti-tuberculous drugs with successful outcome.
\end{abstract}

Keywords: Tuberculosis, abdominal tuberculosis, extrapulmonary, gastrointestinal, rectal.

\section{Introduction}

Tuberculosis continues to be a major source of morbidity and mortality in Bangladesh. Gastrointestinal (GI) tract is the sixth commonest site for extrapulmonary tuberculosis, comprising only $3 \%-4 \%$ of all extrapulmonary involvement. ${ }^{1}$ Approximately $20 \%-25 \%$ of cases of tuberculosis involving the GI tract have simultaneous pulmonary disease. ${ }^{1}$ Colonic tuberculosis with isolated involvement of anorectum is even rarer. ${ }^{2}$ As presentation of rectal tuberculosis may mimic malignancy, it is imperative to arrive at a diagnosis early as anti-tuberculous (anti-TB) drugs are very effective and surgical intervention for the patient can be avoided.

We present two cases of rectal tuberculosis with different clinical manifestations and colonoscopic findings. Our cases demonstrate the importance of keeping the diagnosis of isolated rectal tuberculosis in mind to avoid pitfalls in managing such cases.

\section{Case reports}

Case 1

A 50 years old female presented with a history of constipation, passage of mucus per rectum and gradual weight loss over a period of 3 months. She had no history of bleeding per rectum, altered bowel habits, evening rise of temperature, cough, hemoptysis or contact history of tuberculosis.

General examination of the patient was unremarkable. Examination of respiratory, cardiovascular and abdomen revealed no abnormality. Rectal examination showed ulcerated growth with raised everted margin about $8 \mathrm{~cm}$ from anal verge without any stricture. The complete blood count was normal except the erythrocyte sedimentation rate (ESR) which was $75 \mathrm{~mm}$ in $1^{\text {st }}$ hour. Sputum for acid-fast bacillus (AFB) was negative. The lactate dehydrogenase $(\mathrm{LDH})$ and carcinoembryonic antigen (CEA) levels were normal. Chest X-ray and ultrasonogram of whole abdomen were normal. Colonoscopy showed an ulcerated lesion with elevated and irregular surface in the rectum at $8 \mathrm{~cm}$ from anal verge (Figure 1 ). There is

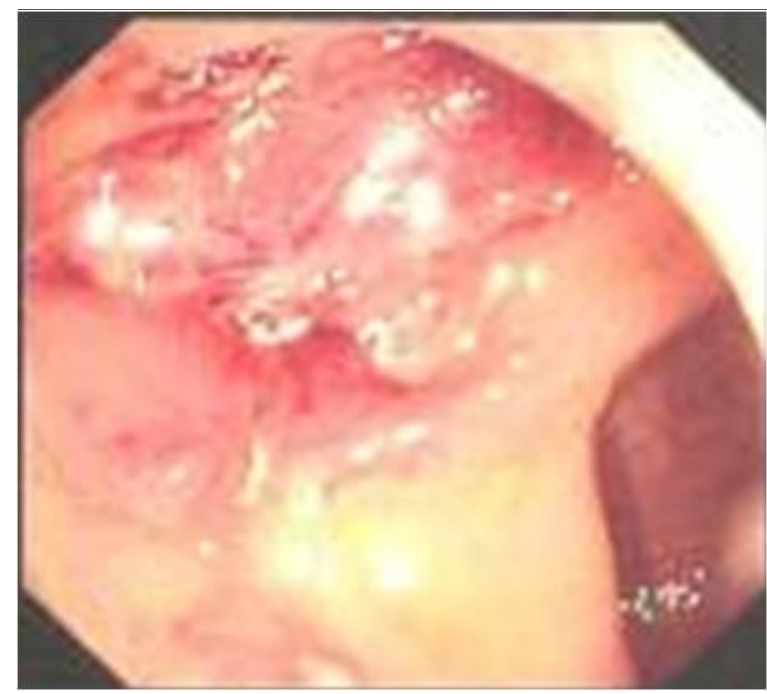

Fig.-1: Colonoscopy showing an ulcerated lesion with elevated and irregular surface in the rectum

1. Professor, Department of Surgery, BSMMU, Dhaka

2. Postgraduate trainee, Department of Medicine, Dhaka Medical College Hospital, Dhaka

3. Assistant Professor, Department of Medicine, Dhaka Medical College, Dhaka

4. Associate Professor, Department of Medicine, Dhaka Medical College, Dhaka

5. Professor, Department of Surgery, Bangladesh Medical College, Dhaka

6. Professor, Department of Medicine, Dhaka Medical College, Dhaka

Correspondence: Prof HAM Nazmul Ahasan, Professor and Head, Department of Medicine, Dhaka Medical College, Dhaka, E-mail: editorjom@yahoo.com 
no luminal narrowing at the site of the lesion. Rest of the colon was normal. Biopsy was taken from edge of the ulcerated area and histopathology showed epitheloid granuloma suggestive of tuberculosis. No evidence of malignancy was seen. The patient was started on anti-TB drugs and was symptom free at follow-up after six months. Repeat colonoscopy was also normal (Figure 2).

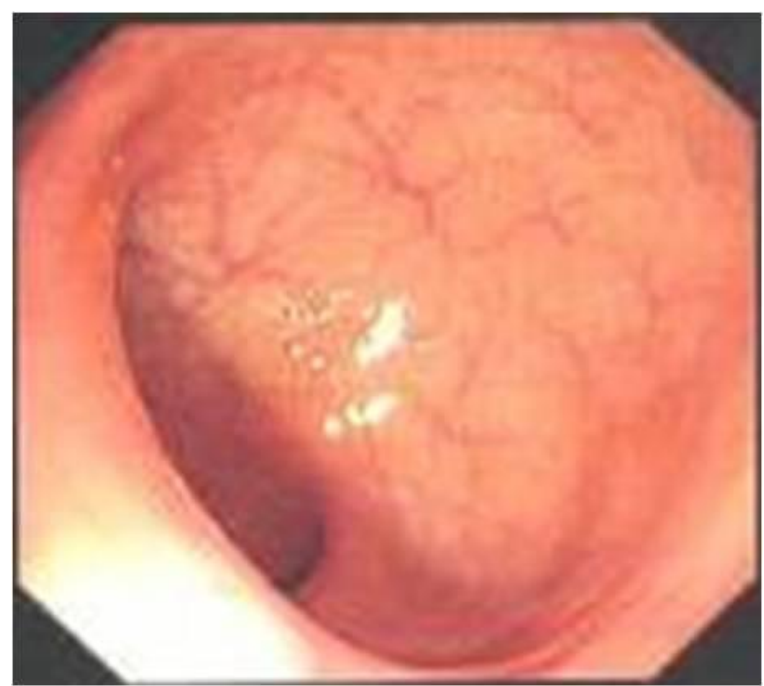

Fig.-2: Repeat colonoscopy of patient 1 showing normal findings

Case 2

A 48 years old male presented with a history of pain and burning sensation after defecation in the anal region after defecation. He had twice undergone fistulotomy two years back. There was no history of bleeding or purulent discharge per rectum, altered bowel habits, cough, hemoptysis, fever or weight loss. He denied any past history of tuberculosis or contact with tuberculosis patient.

General and systemic examination of the patient was normal. Rectal examination showed a hard indurated mass in posterior rectal wall about $3 \mathrm{~cm}$ from anal verge measuring about $2.5 \mathrm{~cm}-\mathrm{by}-2 \mathrm{~cm}$. Complete blood count was within normal limit. CEA level was also normal. Chest X-ray returned normal and sputum for AFB was negative. MRI of pelvis was normal. Flexible sigmoidoscopy showed an indurated area in posterior wall of rectum (Figure 3). A segment of tissue was excised from edge of indurated area and sent for histopathological examination. Histology showed Langhan's giant cells granuloma with early caseation necrosis suggestive of tuberculosis. AntiTB drugs were started and the patient remained asymptomatic at follow-up after six months.

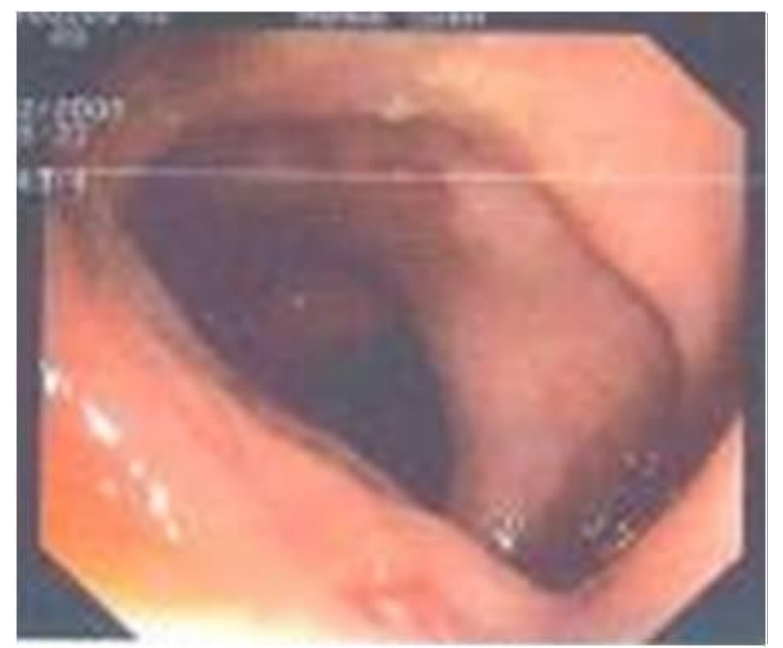

Fig.-3: Flexible sigmoidoscopy showing an indurated area in posterior wall of rectum

\section{Discussion}

Tuberculosis involving colon is a rare extrapulmonary form of the disease, accounting only $3 \%$ $4 \%$ of intestinal tuberculosis. ${ }^{1}$ Within the colon, anorectal region is an unusual site for tuberculosis. In a series of 37 patients described by Mukewar S et al, only $5 \%$ patients had involvement of the rectum. ${ }^{2}$ Although, commonly associated with pulmonary disease, isolated rectal tuberculosis has been reported in a series of 8 patients by Puri et al. ${ }^{3}$

These patients may present with pain in anorectal region, recurrent fistula-in-ano, hematochezia, purulent discharge per rectum, constipation, diarrhea or constitutional symptoms. ${ }^{4}$ Bleeding per rectum in rectal tuberculosis is found in only $3 \%-4 \%$ of cases while constipation can be seen in as much as $37 \%$ of cases. ${ }^{5}$ Clinical and procto-sigmoidoscopic examination may reveal nodularity, ulceration, tight strictures, recto-vesical or recto-urethral fistula. ${ }^{6}$ Majority of cases investigated by Mukewar $\mathrm{S}$ et al showed either ulceration (92\%) or nodule (54\%) on colonoscopy. ${ }^{2}$ Strictures often develop in anorectal region and are usually situated within $10 \mathrm{~cm}$ of anal verge. Fibrosis in this region may lead to an increase of presacral space which can be detected by MRI. ${ }^{7}$ Rectal tuberculosis should be considered as a diagnostic possibility in patients presenting with one or more of these features. To confirm presence of tuberculosis and to exclude malignancy, biopsy and histological examination is mandatory. Co-existence of tuberculosis and adenocarcinoma in rectum has been described although this association remains 
controversial. ${ }^{8}$ Histological examination demonstrates features of chronic granulomatous inflammation with caseation necrosis. Repeated biopsies may be required in some cases as tuberculous lesions may be submucosal in nature. ${ }^{6}$ Demonstration of AFB in biopsy specimen or culture is the most specific investigation although diagnostic yield in either procedure is usually low. ${ }^{4}$ Polymerase chain reaction is an option in cases where AFB are not found in biopsy or culture returns negative.

Tuberculosis of rectum can be treated successfully with standard anti-TB drug regimen. Surgery may be required in those who have failed medical management. Delayed surgical intervention following initial medical management reduces complications significantly. ${ }^{3,6}$

The two cases presented here highlight the need to recognize the possibility of tuberculosis in an endemic region for TB like Bangladesh. Low index of suspicion can lead to delay in diagnosis, unnecessary invasive procedures and repeated surgical interventions raising the possibility of stricture formation or anal incontinence. In conclusion, tuberculosis in rectum should be strongly considered as differential diagnosis in patient presenting with rectal mass keeping in mind that the prognosis with anti-TB treatment is very satisfactory, sparing the patient of unwarranted surgical intervention unless complications develop.

\section{References}

1. Chong V H, Lim K S. Gastrointestinal tuberculosis. Singapore Med J 2009; 50: 638-646

2. Mukewar S, Mukewar S, Dua KS. Tuberculosis of the colon: endoscopic features with prospective follow-up after anti-tuberculosis treatment. Gastrointest Endosc 2007;65: AB253.

3. Puri AS, Vij JC, Chaudhary A, et al. Diagnosis and outcome of isolated rectal tuberculosis. Dis Colon Rectum. 1996;39:1126-1129.

4. Samarasekera DN, Nanayakkara PR. Rectal tuberculosis: A rare cause of recurrent rectal suppuration. Colorectal Dis 2008;10:846-847.

5. Patankar T, Babulkar J, Prasad S, et al. Isolated rectal tuberculosis masquerading as malignancy. Bombay Hosp J 2009;51(02)

6. Rasheed S, Zinicola R, Watson D, et al. Intraabdominal and gastrointestinal tuberculosis. Colorectal Dis 2007;9:773-783.

7. Sharma MP, Bhatia V. Abdominal tuberculosis. Indian J Med Res 2004;120:305-315.

8. Khaniya S, Koirala R, Shakya VC, et al. Anorectal tuberculosis coexisting with adenocarcinoma: an unusual association. Cases Journal 2009;2:143. 